\title{
La combinación de docetaxel y capsaicina inhibe sinérgicamente el crecimiento de células de cáncer de próstata.
}

\author{
Belén Sánchez Gómeza ${ }^{\mathrm{a}}$, Alicia Bort ${ }^{\mathrm{b}}$, Pedro Mateos-Gómez ${ }^{\mathrm{c}}$, Irene de Migueld, \\ Nieves Rodríguez-Henche ${ }^{e}$, Inés Díaz-Laviada ${ }^{f}$ \\ Departamento de Biología de Sistemas, Universidad de Alcalá. 28871, Alcalá de Henares, E-28871, Madrid, España.
}

a. belensg.88@gmail.com b. aliciabort@gmail.com c.pedroantonio.mateos@uah.es

d. irenedemiguelgarcia@gmail.com e.nieves.rhenche@uah.es f. ines.laviada@uah.es

IV Congreso de Señalización Celular, SECUAH 2019.

20-22 de marzo, 2019. Universidad de Alcalá. Alcalá de Henares, Madrid. España

Sesión de paneles.

Palabras clave: Docetaxel; capsaicina; sinergia; células PC3; células LNCaP: cáncer de próstata

\section{Resumen}

La quimioterapia actual para el cáncer de próstata resistente a la castración se basa en compuestos derivados de taxanos como el docetaxel. Sin embargo, la aparición de resistencia y efectos secundarios limita el beneficio terapéutico, siendo la principal preocupación en el tratamiento del cáncer de próstata. Las terapias combinadas en muchos casos mejoran la eficacia del fármaco y retrasan la aparición de efectos no deseados, lo que constituye una alternativa para el tratamiento del cáncer de próstata resistente a la castración. En este estudio, comprobamos la eficacia del tratamiento combinado de docetaxel y capsaicina, el compuesto responsable del sabor picante de los pimientos, en la proliferación de células de cáncer de próstata. Los resultados obtenidos mostraron que el tratamiento combinado de docetaxel y capsaicina inhibió sinérgicamente el crecimiento de las células LNCaP y PC3, con un índice de combinación inferior a 1 para la mayoría de las combinaciones probadas. Además, el tratamiento combinado disminuyó notablemente la fosforilación de Akt y de sus dianas mTOR y S6, situadas por debajo en su cascada de señalización. Por otro lado, la disminución de la expresión de Akt impidió el efecto antiproliferativo del docetaxel, mientras que potenció el de la capsaicina. Todo esto sugiere que el efecto antiproliferativo sinérgico podría atribuirse a la inhibición de la vía de señalización PI3K/Akt/mTOR. A su vez, los experimentos in vivo confirmaron el efecto sinérgico del docetaxel y la capsaicina para reducir el crecimiento tumoral de células PC3 en ratones inmunodeficientes. Por tanto, nuestros resultados indican que el tratamiento combinado de docetaxel y capsaicina representa un enfoque terapéutico relevante para el tratamiento del cáncer de próstata.

Cita: Sánchez Gómez, Belén; Bort, Alicia; Mateos-Gómez, Pedro; de Miguel, Irene; Rodríguez-Henche, Nieves; Díaz-Laviada, Inés (2019) La combinación de docetaxel y capsaicina inhibe sinérgicamente el crecimiento de células de cáncer de próstata. Actas del IV Congreso de Señalización Celular, SECUAH 2019. 20-22 de marzo, 2019. Universidad de Alcalá. Alcalá de Henares, Madrid. España. Sesión de paneles. dianas 8 (1): e201903fa04. ISSN 1886-8746 (electronic) journal.dianas.e201903fa04 http://www3.uah.es/dianas?e201903fa04. URI http://hdl.handle.net/10017/15181

Copyright: @ Sánchez-Gómez B, Bort A, Mateos-Gómez P, de-Miguel I, Rodríguez-Henche N, Díaz-Laviada I. Algunos derechos reservados. Este es un artículo open-access distribuido bajo los términos de una licencia de Creative Commons Reconocimiento-NoComercial-SinObraDerivada 4.0 Internacional. http://creativecommons.org/licenses/by-nc-nd/4.0/

\section{Introducción}

El cáncer de próstata $(\mathrm{PCa})$ es la neoplasia maligna más prevalente en los hombres a nivel mundial y la segunda causa de muerte por cáncer $[1,2]$. Los factores ambientales, como las dietas hipercalóricas, el sedentarismo, el aumento de la esperanza de vida y los avances en las técnicas de diagnóstico han contribuido al aumento de la incidencia del PCa. Para los cánceres localmente avanzados y metastásicos, se recurre a la terapia de privación de andrógenos (ADT) o castración química. No obstante; a pesar de la regresión inicial de la enfermedad, el tumor puede llegar a adquirir resistencia a la terapia y comenzar a progresar. Este estado se conoce como PCa resistente a la castración (CRPC) y se asocia con bajas tasas de supervivencia, mayor agresividad y peor pronóstico [3].

Actualmente, el docetaxel (DTX) es el agente quimioterapéutico de primera línea para los pacientes con esta forma letal de la enfermedad, pero la supervivencia de los pacientes sigue limitada por la aparición de efectos adversos dependientes de la dosis y la resistencia adquirida. Los mecanismos que sustentan la aparición de resistencia (Fig.1) incluyen la sobreexpresión de bombas de flujo de múltiples fármacos, la mutación de la proteína $\beta$-tubulina y la sobreactivación de proteínas de señalización como MAPK o Akt [4]. La resistencia al DTX es un problema clínico, ya que es la terapia principal para el CRPC. Además, los nuevos fármacos quimioterapéuticos desarrollados para tratar a los pacientes resistentes al DTX tienen 
toxicidades hematológicas significativas [4]. Por tanto, se necesitan nuevas estrategias terapéuticas para mejorar la quimioterapia basada en taxanos [5]. Entre estas estrategias, cobra gran importancia clínica la identificación de agentes que, cuando se combinen con los fármacos quimioterapéuticos actuales, permitan disminuir las dosis sin reducir su eficacia, así como evitar y/o superar la resistencia al fármaco. De esta manera, la terapia combinada, una modalidad de tratamiento que combina dos o más agentes terapéuticos, se está convirtiendo en una alternativa clave en la terapia contra el cáncer [6].

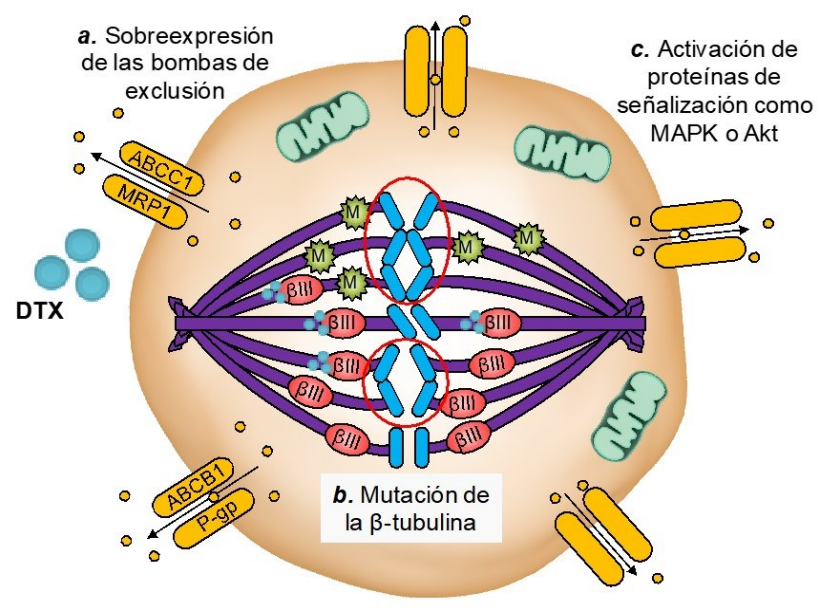

Figura 1.- Mecanismos implicados en la aparición de resistencia al DTX en PCa. Se representan algunos: (a) sobreexpresión de bombas de flujo de múltiples fármacos, (b) mutación de la proteína $\beta$ tubulina y (c) sobreactivación de proteínas de señalización como MAPK o Akt.

En los últimos años, se han obtenido muchos medicamentos anticancerígenos a partir de compuestos naturales. La capsaicina (CAP), el ingrediente picante de los pimientos picantes, muestra actividad antineoplásica en muchas líneas celulares de cáncer, así como in vivo [7]. Además, los datos recientes indican que la CAP sensibiliza las células a los agentes quimioterapéuticos. Por ejemplo, la combinación de CAP y camptotecina aumenta la apoptosis en cáncer de pulmón de células pequeñas [8]. En el colangiocarcinoma, la CAP aumenta la sensibilidad al 5-fluorouracilo y la mezcla de ambos compuestos inhibe el crecimiento del tumor con mayor eficacia que el 5-fluorouracilo [9]. En células de PCa humano, la CAP combinada con brasinina potencia los efectos apoptóticos y antimetastásicos [10]. También hemos demostrado que, en las células de hepatocarcinoma, la CAP aumenta los efectos antiproliferativos del sorafenib [11]. Sin embargo, los mecanismos por los que la CAP inhibe la proliferación celular y sensibiliza las células a los fármacos, son diversos y poco conocidos.

La vía de señalización $\mathrm{PI} 3 \mathrm{~K} / \mathrm{Akt} / \mathrm{mTOR}$, regula distintos procesos celulares como metabolismo, supervivencia, proliferación, apoptosis, crecimiento y migración celular. Las PI3K de clase I fosforilan fosfatidilinositol-4,5-bifosfato $\left(\mathrm{PIP}_{2}\right)$ generando el fosfatidilinositol-3,4,5-trifosfato $\left(\mathrm{PIP}_{3}\right)$. Este último actúa como segundo mensajero activando la proteína PDK que a su vez fosforila Akt activándola. Para su máxima activación Akt requiere además la fosforilación por mTORC2. Mediante fosforilación, Akt activa proteínas involucradas en el proceso de migración e inhibe proteínas pro-apoptóticas [12]. Debido a la amplia gama de procesos celulares en los que la proteína Akt está involucrada, su desregulación se asocia con la progresión del cáncer. Más de la mitad de los cánceres de próstata en estadio avanzado se produce por una deleción del gen supresor de tumores PTEN, que regula negativamente la vía PI3K/Akt/mTOR, por lo que esta vía se encuentra constitutivamente activa [13].

En este estudio, evaluamos la capacidad de la CAP para inhibir la proliferación de células de PCa. Encontramos que CAP sinergiza con DTX para bloquear potentemente el crecimiento celular in vitro y el crecimiento tumoral in vivo posiblemente por la inhibición de la vía PI3K/Akt/mTOR.

\section{Materiales y Métodos}

\section{Materiales}

La capsaicina (CAP) y el docetaxel (DTX) se compraron a TOCRIS (Bristol, Reino Unido). Los anticuerpos primarios contra las formas totales y fosforiladas de Akt-ser473, mTOR y S6 se obtuvieron de Cell Signaling Technology (Danvers, MA, EE. UU.). La IgG anti-ratón secundaria marcada con peroxidasa era de Sigma (St. Louis, MO, EE. UU.) y la IgG anti-conejo de Calbiochem (San Diego, EE. UU.).

\section{Cultivo de células}

Las líneas celulares de cáncer de próstata humano PC3 y LNCaP se obtuvieron de American Type Culture Collection (ATCC CRL-1435 y ATCC CRL-1740 respectivamente) (Rockville, MD, EE. UU.). 
Las células se cultivaron de forma rutinaria en medio RPMI 1640 suplementado con $100 \mathrm{UI} / \mathrm{ml}$ de penicilina $\mathrm{G}$ sódica, $100 \mu \mathrm{g} / \mathrm{ml}$ de sulfato de estreptomicina, $0,25 \mu \mathrm{g} / \mathrm{ml}$ de anfotericina B (Invitrogen, Paisley, Reino Unido) y 10\% de suero bovino fetal. Para los experimentos de tratamiento, las células se sembraron en placas y se cultivaron durante $48 \mathrm{~h}$, el medio se reemplazó por RPMI 1640 sin suero durante 24 horas y luego se incubaron con diferentes tratamientos durante los tiempos indicados.

\section{MTT}

La viabilidad celular se midió mediante el ensayo de proliferación celular de MTT (3-(4,5-dimetiltiazol2-il)-2,5-difeniltetrazolio) (Sigma, St. Louis, MO, EE. UU.) 24 h después de la exposición a los tratamientos. Se sembraron un total de 5000 células/pocillo en una placa de 12 pocillos en un volumen final de $1 \mathrm{ml}$. Después de los tratamientos, se añadieron $100 \mu \mathrm{l}$ de solución de MTT $(5 \mathrm{mg} / \mathrm{ml}$ en PBS $)$ al medio y las células se incubaron a $37^{\circ} \mathrm{C}$ durante $4 \mathrm{~h}$. Luego, el sobrenadante se desechó y se añadió dimetilsulfóxido para disolver los cristales de formazán. Los tratamientos se realizaron por triplicado. La densidad óptica de cada pocillo se evaluó mediante la medición de la absorbancia a 490 y $650 \mathrm{~nm}$ utilizando un lector de absorbancia iMark ${ }^{\mathrm{TM}}$ de Bio-Rad (Richmond, CA, EE. UU.).

\section{Western-blot}

Las células se lisaron en tampón de lisis que contenía inhibidor de proteasas e inhibidor de fosfatasas (Roche, Diagnostics; Mannheim, Alemania), se incubaron en hielo durante 15 min y se aclararon por microcentrifugación. Las concentraciones de proteínas se midieron con el kit de ensayo de proteínas BioRad $^{\mathrm{TM}}$ (Richmond, CA, EE. UU.). Los extractos de proteínas celulares se separaron por PAGE-SDS, detectándose a continuación mediante Western-blot [11].

\section{Silenciamiento mediante RNA de interferencia (siRNA)}

La transfección se llevó a cabo utilizando $1 \mathrm{ml}$ de OptiMEM que contenía $4 \mu \mathrm{g}$ de lipofectamina iMax (Invitrogen, Carlsbad, CA), con RNA de interferencia $100 \mathrm{nM}$ específico para Akt o inespecífico de control ( $\mathrm{siC}$ ) durante $72 \mathrm{~h}$ según los protocolos del fabricante (Invitrogen, Carlsbad, CA). A las 72 horas después de la transfección, el medio se eliminó y se reemplazó por RPMI que contenía un 10\% de suero fetal bovino. Tras la transfección, las células se usaron para ensayos de viabilidad celular MTT o Western-blot.

\section{Actividad antitumoral en modelos de ratón}

Se compraron ratones atímicos nude-Foxn1 (nu/nu) de 4 semanas de edad de Envigo RMS (Barcelona, España) y se alojaron en un gabinete de flujo de aire laminar en condiciones libres de patógenos en un horario de $12 \mathrm{~h}$ de luz/oscuridad a $21-23^{\circ} \mathrm{C}$ y $40-60 \%$ de humedad con acceso a gránulos de alimentos y agua del grifo a voluntad. 4 animales fueron alojados por jaula. Se utilizó el análisis de potencia de G para calcular el tamaño de la muestra, de acuerdo con nuestros datos y experiencia anteriores y considerando el efecto de dos colas y un nivel de significación del 5\%. Según el experimento, se indujeron tumores de próstata en ratones atímicos mediante inyección subcutánea de $5 \times 10^{6}$ células PC3. Cuando los tumores alcanzaron los $70 \mathrm{~mm}^{3}$, los ratones se dividieron aleatoriamente en cuatro grupos experimentales de 6 animales cada uno, y los siguientes tratamientos se iniciaron diariamente por inyección intraperitoneal: vehículo (DMSO), $2 \mathrm{mg} / \mathrm{Kg}$ de capsaicina (CAP), $10 \mathrm{mg} / \mathrm{Kg}$ de docetaxel (DTX) o $2 \mathrm{mg} / \mathrm{Kg}$ de capsaicina $+10 \mathrm{mg} / \mathrm{Kg}$ de docetaxel (CAP + DTX). Los tamaños de los tumores se midieron cada día y se calcularon utilizando la fórmula $\mathrm{V}\left(\mathrm{mm}^{3}\right)=1 / 2\left(\right.$ Longitud $\left.\times \mathrm{Ancho}^{2}\right)$. Al final del estudio, los ratones se sacrificaron colocándolos en una cámara llena de gas $\mathrm{CO}_{2}$, y los tumores extirpados se recuperaron y pesaron.

\section{Análisis de drogas combinadas}

La interacción del fármaco se determinó utilizando la ecuación de isobolograma del índice de combinación (CI) que permite la determinación cuantitativa de las interacciones del fármaco, en la que CI $<1$ implica sinergismo, $\mathrm{CI}=1$ efecto aditivo y $\mathrm{CI}>1$ antagonismo implícito. Se usó el software Compusyn versión 1.0 (ComboSyn, Inc. Paramus, NJ, EE. UU.) para generar las curvas de dosisrespuesta, el análisis dosis-efecto y el gráfico del efecto del CI.

\section{Análisis estadístico}

El análisis estadístico de los resultados se realizó mediante ANOVA de dos vías y la prueba de comparaciones múltiples de Dunnett o la prueba de comparaciones múltiples de Tukey. Los resultados se informaron como media \pm S.E.M. o S.D. como se indica en el título de la figura, de al menos tres experimentos independientes. Los datos se consideraron significativos cuando $\mathrm{P} \leqslant 0.05$. 

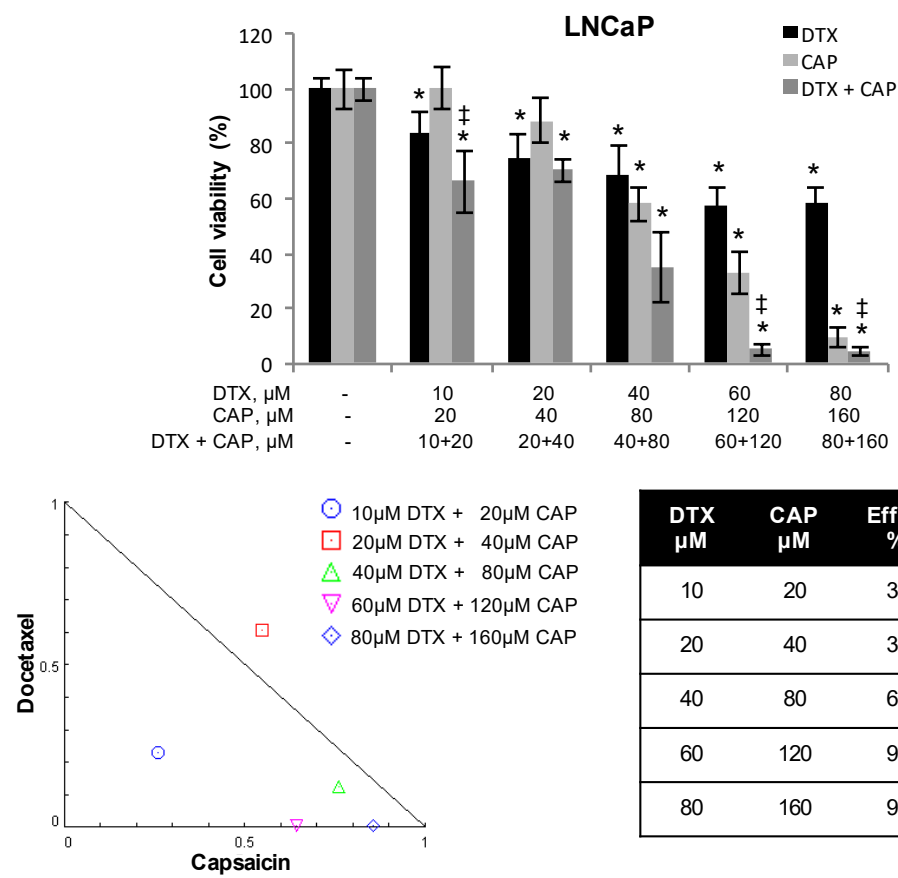

\begin{tabular}{|cccc|}
\hline $\begin{array}{c}\text { DTX } \\
\boldsymbol{\mu M}\end{array}$ & $\begin{array}{c}\text { CAP } \\
\boldsymbol{\mu M}\end{array}$ & $\begin{array}{c}\text { Effect } \\
\%\end{array}$ & $\mathbf{C I}$ \\
\hline 10 & 20 & 34 & 0.490 \\
\hline 20 & 40 & 30 & 1.156 \\
\hline 40 & 80 & 65 & 0.889 \\
\hline 60 & 120 & 95 & 0.649 \\
\hline 80 & 160 & 95 & 0.865 \\
\hline
\end{tabular}
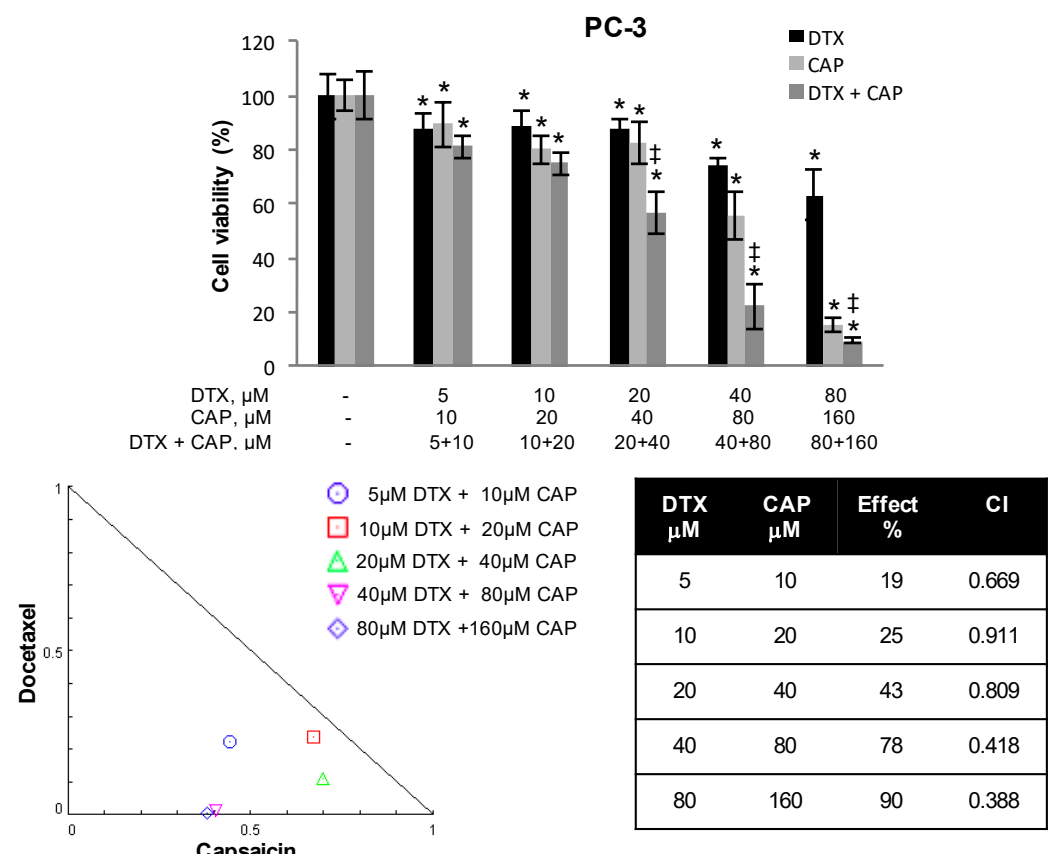

Figura 2.- Efecto antiproliferativo sinérgico de capsaicina y docetaxel en células tumorales de próstata. Las células LNCaP (panel superior) o las células PC3 (panel inferior) se incubaron con dosis crecientes de docetaxel (DTX), capsaicina (CAP) o la combinación de ambos compuestos durante 24 horas y la viabilidad celular se evaluó mediante MTT. Los datos representan la media $(\mathrm{n}=3) \pm \mathrm{SD} .{ }^{*} \mathrm{p}<0,0001$ diferencia significativa entre las células tratadas y las células control por ANOVA de dos vías y la prueba de comparaciones múltiples de Dunnett; $\$ p<0,0001$ indica una interacción significativa entre el tratamiento con DTX y CAP. Los datos se analizaron con el software CompuSyn para calcular el índice de combinación (CI).

\section{Resultados}

\section{La capsaicina y el docetaxel inhiben sinérgicamente el crecimiento de las células de $\mathrm{PCa}$}

Para evaluar el efecto antiproliferativo del docetaxel (DTX) y la capsaicina (CAP), se incubaron células de cáncer de próstata LNCaP y PC3 con dosis crecientes de DTX, CAP o la combinación de ambos compuestos y la viabilidad celular se determinó mediante MTT. Como se muestra en la Fig. 2, tanto DTX como CAP reducen individualmente la viabilidad de las células LNCaP y PC3. La CAP sola inhibió la viabilidad celular de forma dependiente de la dosis desde $40 \mu \mathrm{M}$ en células LNCaP y desde $20 \mu \mathrm{M}$ en células PC3. Cabe destacar que la concentración inhibitoria del 50\% (CI50) fue mucho más alta para DTX $(300 \mu \mathrm{M})$ que para CAP $(72 \mu \mathrm{M})$ en células PC3. Cuando se combinaron DTX y CAP, se observó una reducción dramática en la viabilidad celular. Probamos una relación CAP:DTX constante $(2: 1)$ para crear isobologramas utilizando el software CompuSyn (C) y para calcular el índice de combinación $(\mathrm{CI})$. El CI permite la cuantificación de sinergismo o antagonismo para dos fármacos, donde un CI de 1 indica un 
efecto aditivo, mientras que un $\mathrm{CI}<1$ o $\mathrm{CI}>1$ indica sinergismo o antagonismo, respectivamente. El CI mostró un potente efecto sinérgico sobre la viabilidad celular en cuatro de las cinco combinaciones utilizadas en las células LNCaP y en las cinco combinaciones utilizadas en las células PC3. Del mismo modo, el isobolograma para la combinación de docetaxel y capsaicina mostró que todos los puntos de datos combinados en las células PC3 caen en la parte inferior izquierda, lo que indica sinergismo (Fig. 2). En LNCaP, el efecto sinérgico fue evidente en cuatro combinaciones, pero fue menos fuerte que en las células PC3. Luego, para experimentos adicionales, elegimos la combinación de DTX $40 \mu \mathrm{M}+\mathrm{CAP} 80$ $\mu \mathrm{M}$, ya que tenía un alto efecto sinérgico pero un efecto citotóxico moderado.
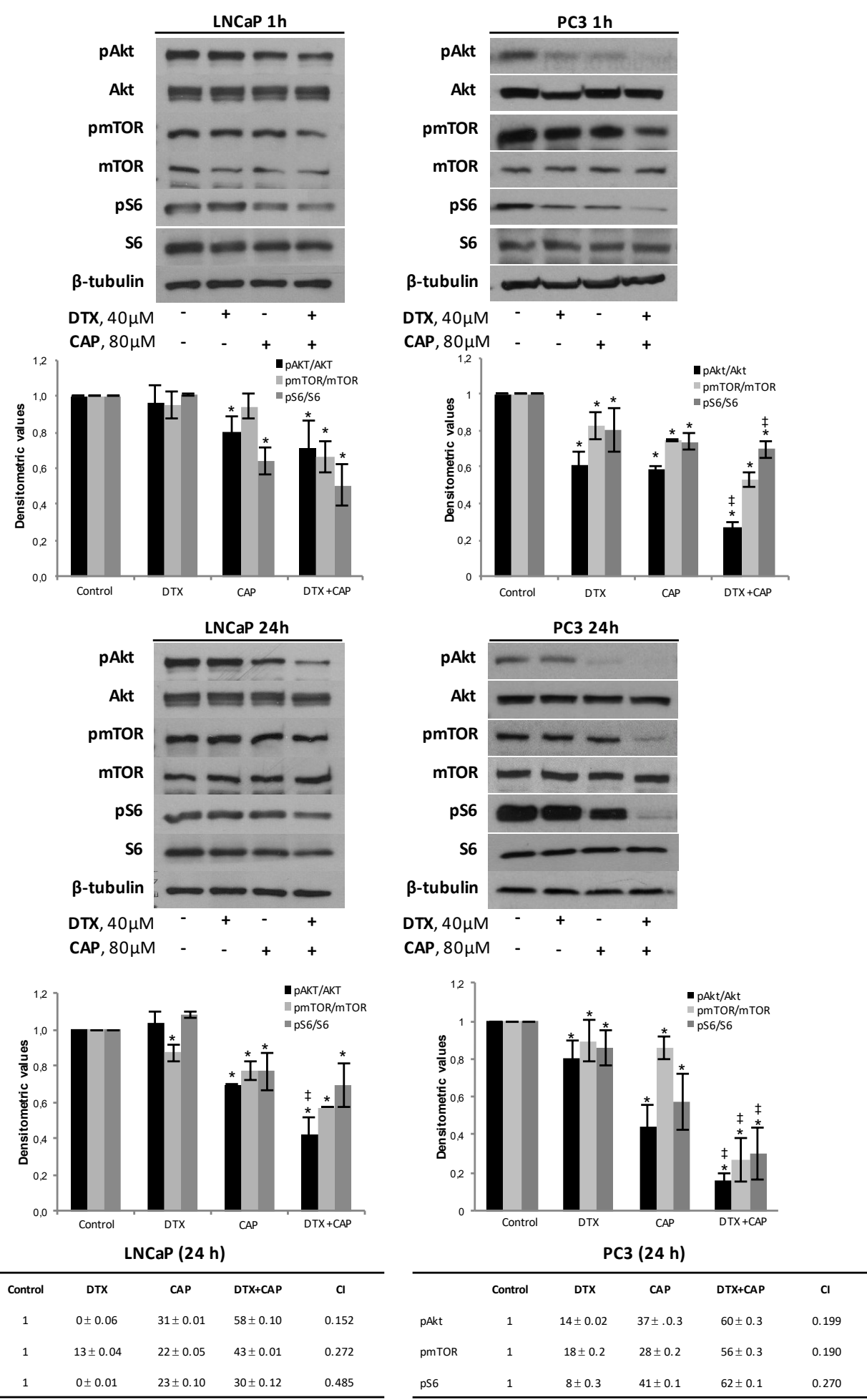

Figura 3.- La combinación de docetaxel y capsaicina inhibe eficazmente la vía de Akt/mTOR. Las células LNCaP o PC3 se incubaron con docetaxel $40 \mu \mathrm{M}$ (DTX), capsaicina $80 \mu \mathrm{M}$ (CAP) o la combinación de ambos durante 1 o 24 horas y los niveles de fosforilación de Akt, mTOR, S6 y sus correspondientes formas totales fueron determinados por Western-blot. La $\beta$-tubulina sirve como control de carga. Los análisis densitométricos de las bandas representadas como la media \pm desviación estándar de tres experimentos diferentes se muestran debajo de los gráficos. $* \mathrm{p}<0,05$ diferencia significativa entre las células tratadas y las células de control por ANOVA de dos vías y la prueba de comparaciones múltiples de Dunnett; $\mathrm{y} \ddagger \mathrm{p}<0.05$ indican una interacción significativa entre el tratamiento con DTX y CAP. 


\section{La capsaicina y el docetaxel inhiben la vía PI3K/Akt/mTOR}

Akt es el componente principal de la ruta de señalización PI3K/Akt/mTOR que está constitutivamente activa en las células PC3 y LNCaP debido a una deleción del gen supresor de tumores PTEN. La activación de esta vía de señalización está involucrada en muchas funciones que inducen el crecimiento celular. Para corroborar si el efecto antiproliferativo del tratamiento combinado de DTX y CAP estaba relacionado con esta vía, evaluamos la fosforilación de Akt y de sus efectores posteriores mTOR y S6.

Los resultados mostraron que el DTX indujo una inhibición moderada de la fosforilación de Akt, mTOR y S6 solo en las células PC3 y que la CAP disminuyó la fosforilación de Akt, mTOR y S6 tanto en las células PC3 como en las LNCaP a la 1 y a las 24 h (Figura 3). Sin embargo, el tratamiento conjunto con DTX y CAP condujo a una mayor inhibición de la fosforilación de Akt en células LNCaP y suprimió la fosforilación de Akt en células PC3, tanto a la hora como a las 24 horas (Fig. 3). Del mismo modo, la fosforilación de mTOR y S6 también se inhibieron significativamente en las células PC3 tratadas conjuntamente (Fig. 3). En las células LNCaP, el tratamiento combinado también causó la inhibición de la fosforilación de mTOR y S6, pero el efecto fue más débil que el de las células PC3 incluso a las 24 h, lo que indica que el tratamiento combinado es más eficaz en las células de cáncer de próstata PC3 resistentes a la castración (Fig. 3). El análisis ANOVA reveló que el efecto inhibitorio producido por $\mathrm{DTX}+\mathrm{CAP}$ en la fosforilación de Akt, mTOR y S6 era diferente del producido por DTX o CAP dado individualmente. Además, el efecto inhibitorio sobre Akt, mTOR y S6 a las 24 h en las células tratadas fue mayor que la suma de los efectos individuales de DTX y CAP y el índice de combinación fue menor que 1, lo que indica sinergismo (Fig. 3). Esos resultados sugieren que CAP y DTX bloquean de forma sinérgica la ruta $\mathrm{PI} 3 \mathrm{~K} / \mathrm{Akt} / \mathrm{mTOR}$, lo que podría subyacer al efecto antiproliferativo de la combinación de DTX y CAP en células de PCa.

Para corroborar esto, silenciamos Akt por RNA de interferencia y determinamos la viabilidad celular por MTT. Como se muestra en la Fig. 4, el bloqueo de la ruta PI3K/Akt/mTOR eliminó el efecto que el DTX ejercía sobre la viabilidad de las células PC3, mientras que potenció el de la CAP. Esto parece indicar que el DTX necesita a Akt para ejercer su efecto antiproliferativo y que la inhibición de la vía PI3K/Akt podría ser la responsable del efecto antiproliferativo sinérgico provocado por la combinación de DTX y CAP en las células de cáncer de próstata PC3 resistentes a la castración.
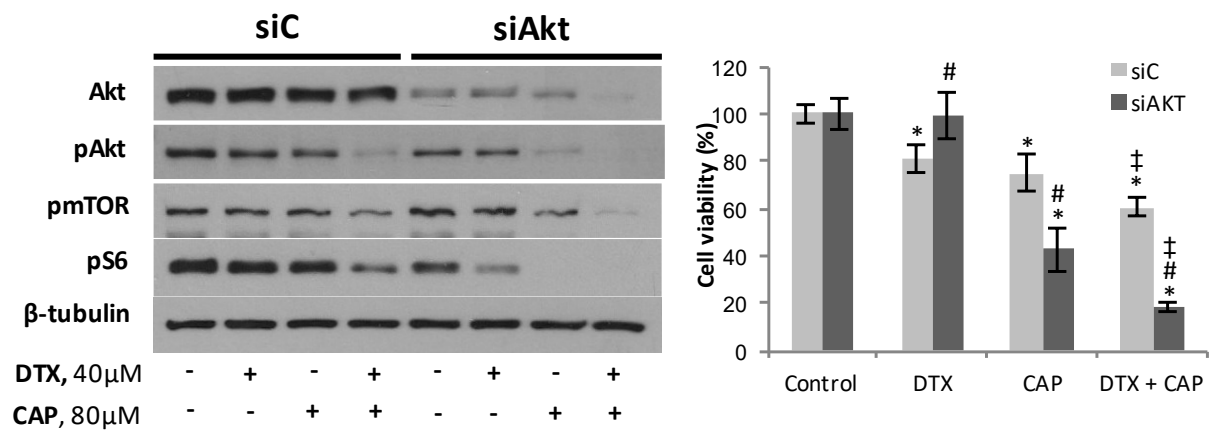

Figura 4.- El bloqueo de la ruta de PI3K/Akt dificulta el efecto inhibitorio del docetaxel en el crecimiento de células de PCa. Akt fue silenciado en las células PC3 por transfección con RNA de interferencia selectivo. Los niveles de fosforilación de Akt, mTOR, S6 y sus correspondientes formas totales determinadas por Western-blot se muestran a la izquierda. La viabilidad celular por MTT se muestra a la derecha.

\section{Estudio antitumoral in vivo}

Para evaluar la eficacia terapéutica de la combinación de DTX y CAP in vivo, se indujeron tumores de xenoinjerto de células PC3 en ratones inmunodeprimidos mediante inyección subcutánea en uno de los flancos.

Cuando los tumores alcanzaron un volumen de $70 \mathrm{~mm}^{3}$, los animales se asignaron al azar a varios grupos $(\mathrm{n}=6)$ y fueron tratados con CAP o DTX por separado o con el tratamiento combinado de ambos compuestos mediante inyección intraperitoneal. A pesar de que 6 días después del inicio del tratamiento el DTX disminuyó significativamente el crecimiento de los tumores, el co-tratamiento de DTX + CAP logró una eficacia antitumoral significativamente mayor. Como se muestra en la Figura 5, la combinación de docetaxel y capsaicina mostró una mayor actividad antitumoral que cualquiera de los agentes por sí solos. Pasados 15 días de tratamiento, el volumen tumoral de los ratones tratados con $2 \mathrm{mg} / \mathrm{kg}$ de CAP se redujo en un 24\% respecto al de los controles, el de los tratados con $10 \mathrm{mg} / \mathrm{kg}$ de DTX se redujo en un $45 \%$ y el de los tratados con el tratamiento combinado sufrió una reducción del 78\% (Fig. 5A). Del mismo modo, los tumores de los ratones tratados con terapia de combinación tuvieron un peso húmedo significativamente menor que el de los tratados con DTX o CAP por separado (Fig. 5B). 
Al final de los 15 días, se analizó el estado de la vía PI3K/Akt/mTOR en los tumores de células PC3. La Figura 5B muestra que, en línea con los resultados in vitro, la actividad de Akt y mTOR en los tumores tratados con el tratamiento combinado fue sustancialmente menor que en los tratados con cada uno de los compuestos por separado. Por tanto, estos resultados indican que la combinación de capsaicina y docetaxel reduce sinérgicamente el crecimiento de los tumores de células PC3 in vivo y es eficaz para este modelo de PCa resistente a la castración.

A

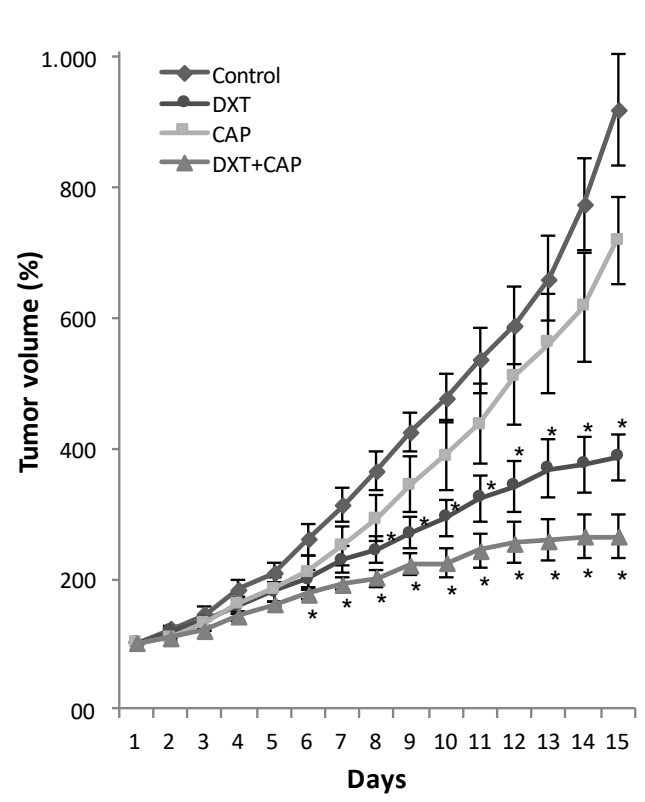

B
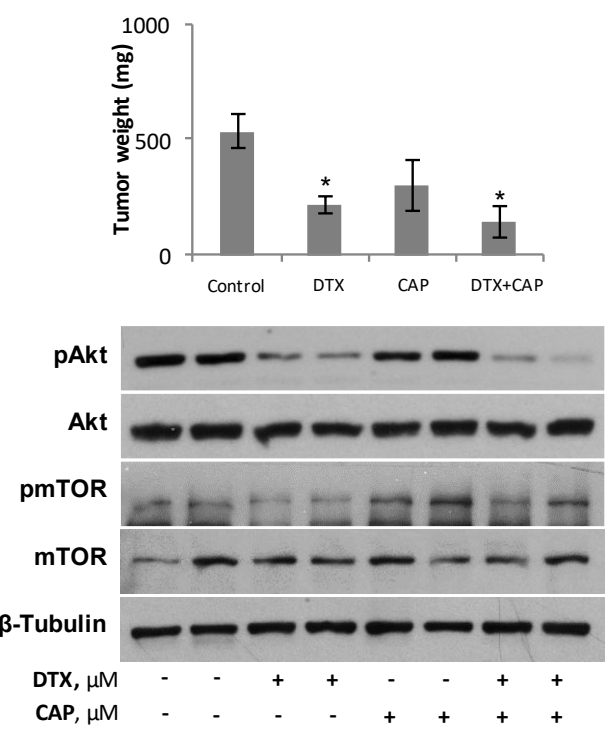

Figura 5.- Actividad antitumoral in vivo de la combinación de docetaxel y capsaicina. Ratones atímicos fueron inyectados subcutáneamente en el flanco derecho con células PC3. Cuando los tumores alcanzaron un tamaño de 70 $\mathrm{mm}^{3}$, los ratones se trataron diariamente mediante inyección intraperitoneal con vehículo (control), $10 \mathrm{mg} / \mathrm{kg} \mathrm{de}$ docetaxel (DTX), $2 \mathrm{mg} / \mathrm{kg}$ de capsaicina (CAP) o ambos compuestos (DTX+CAP). Los volúmenes tumorales se midieron diariamente. A. Curva de crecimiento de tumores de células PC3 después de la administración de vehículo (diamantes), DTX (círculos), CAP (cuadrados) o ambos compuestos (triángulos). Los resultados representan la media \pm SEM de 6 ratones en cada grupo. ${ }^{*} \mathrm{p}<0.05$ diferencia significativa entre los ratones tratados y de control por ANOVA de dos vías y la prueba de comparaciones múltiples de Tukey. A continuación se muestra el peso del tumor al final del tratamiento. B. Peso de los tumores y análisis por Western-blot de la vía PI3K/Akt/mTOR en los tumores disecados de PC3.

\section{Discusión}

El DTX es considerado el fármaco anticancerígeno más prometedor para el tratamiento del PCa. Sin embargo, la rápida aparición de resistencia y toxicidad sistémica disminuyó su eficacia. La terapia de combinación representa una estrategia terapéutica prometedora para superar la toxicidad mediante la reducción de la dosis efectiva. Varios agentes prometedores están emergiendo con un papel potencial en las combinaciones con DTX. Los hallazgos preclínicos sugieren que la combinación de estas estrategias innovadoras con los tratamientos tradicionales ofrece nuevos beneficios que mejoran el resultado del tratamiento [14, 15]. En este estudio evaluamos la efectividad de la combinación de docetaxel y el compuesto natural capsaicina para reducir el crecimiento del tumor de próstata. Encontramos que la combinación de ambos compuestos mostró un efecto antitumoral sinérgico tanto in vitro como in vivo, al igual que se han obtenido resultados similares con otros compuestos utilizados en combinación con DTX [16-18]. Nuestros resultados muestran que la combinación de DTX y CAP causó una fuerte disminución en los niveles de fosforilación de Akt, mTOR y S6 y que el bloqueo de esta vía impide la inhibición de la viabilidad celular inducida por DTX y el efecto sinérgico.

Los datos recientes indican que la CAP presenta sinergismo con diversos fármacos convencionales como la camptotecina [8], la pirarubicina [19], la brasinina [10] y el resveratrol en varias líneas celulares tumorales [20]. Sin embargo, los mecanismos moleculares implicados en este efecto sinérgico siguen siendo en gran parte desconocidos. Resultados previos [11] dilucidaron un importante papel de la vía $\mathrm{PI} 3 \mathrm{~K} / \mathrm{Akt} / \mathrm{mTOR}$ en el comportamiento sinérgico del tratamiento combinado de CAP y sorafenib en hepatocarcinoma. Del mismo modo, nuestros resultados sugieren que la combinación de DTX y CAP ejerce su efecto antiproliferativo mediante la inhibición de la vía PI3K/Akt/mTOR.

Para investigar más a fondo el efecto antitumoral sinérgico de la combinación de DTX y CAP, se indujeron tumores de xenoinjerto en ratones desnudos que se trataron con CAP, DTX o ambos. De acuerdo con los datos publicados sobre la biodisponibilidad y absorción de la capsaicina [21], para los 
estudios in vivo utilizamos una dosis de capsaicina equivalente a la utilizada con las células (considerando un volumen de sangre de ratones de $2,5 \mathrm{ml}$ y un peso medio de ratones de $30 \mathrm{~g}, 80 \mu \mathrm{M}$ es equivalente a 2 $\mathrm{mg} / \mathrm{kg}$ ). Por otro lado, el DTX tiene una baja biodisponibilidad principalmente debido a su escasa solubilidad acuosa y su transporte en la sangre mediante la unión a proteínas plasmáticas como las lipoproteínas, la albúmina y la glicoproteína ácida $\alpha 1$. Por lo tanto, las dosis in vivo de docetaxel son generalmente más altas que las usadas en las células. Por lo tanto, elegimos una dosis de docetaxel de 10 $\mathrm{mg} / \mathrm{kg}$, que es la dosis utilizada comúnmente en los estudios in vivo [22-24]. En los tumores de células PC3, DTX y CAP disminuyeron significativamente el crecimiento del tumor y la combinación de DTX + CAP tuvo una actividad antitumoral más fuerte que cualquiera de los dos compuestos administrados por separado. El tratamiento conjunto indujo una inhibición robusta de la vía PI3K/Akt/mTOR en los tumores de próstata PC3. Por lo tanto, demostramos que la combinación propuesta de DTX y CAP inhibe fuertemente el crecimiento de células de cáncer de próstata resistentes a la castración tanto in vitro como in vivo.

\section{Conclusión}

Estos hallazgos indican que la combinación de capsaicina y docetaxel reduce sinérgicamente el crecimiento de células de cáncer de próstata in vitro e in vivo mediante la inhibición de la vía $\mathrm{PI} 3 \mathrm{~K} / \mathrm{Akt} / \mathrm{mTOR}$. Por tanto, la administración conjunta de docetaxel y capsaicina podría representar una estrategia terapéutica relevante para mejorar la quimioterapia con docetaxel en pacientes con cáncer de próstata.

\section{Agradecimientos}

Los autores desean agradecer a la Fundación Tatiana Pérez de Guzmán el Bueno (Beca $n^{\circ}$ Patrocinio 2013-01; 2019-001) por su apoyo financiero en la investigación.

\section{Bibliografía}

1. Siegel RL, Miller KD, Jemal A. Cancer statistics, 2017. CA Cancer J Clin. 2017;67(1):7-30. doi: $10.3322 /$ caac. 21387

2. Torre LA, Siegel RL, Ward EM, Jemal A. Cancer Epidemiol Biomarkers Prev 25: 16-27.

3. Ban F, Dalal K, LeBlanc E, Morin H, Rennie PS, Cherkasov A. 2018. Cheminformatics Driven Development of Novel Therapies for Drug Resistant Prostate Cancer. Mol Inform. 2016. doi: 10.1002/minf.201800043.

4. Ganju A, Yallapu MM, Khan S, Behrman SW, Chauhan SC, Jaggi M. Nanoways to overcome docetaxel resistance in prostate cancer. Drug Resist Updat. 2014;17(1-2):13-23. doi: 10.1016/j.drup.2014.04.001.

5. Kroon J, Kooijman S, Cho NJ, Storm G, van der Pluijm G. Improving taxane-based chemotherapy in castration-resistant prostate cancer. Trends Pharmacol Sci. 2016;37(6):451-462. doi: 10.1016/j.tips.2016.03.003.

6. Bayat Mokhtari R, Homayouni TS, Baluch N, Morgatskaya E, Kumar S, Das B, Yeger H. Combination therapy in combating cancer. Oncotarget. 2017;8(23):38022-38043.

7. Diaz-Laviada I, Rodriguez-Henche N. The potential antitumor effects of capsaicin. Prog Drug Res. 2014;68:181-208.

8. Friedman JR, Perry HE, Brown KC, Gao Y, Lin J, Stevenson CD, Hurley JD, Nolan NA, Akers AT, Chen YC, et al. Capsaicin synergizes with camptothecin to induce increased apoptosis in human small cell lung cancers via the calpain pathway. Biochem Pharmacol. 2017;129:54-66. doi: 10.1016/j.bcp.2017.01.004.

9. Hong ZF, Zhao WX, Yin ZY, Xie CR, Xu YP, Chi XQ, Zhang S, Wang XM. Capsaicin enhances the drug sensitivity of cholangiocarcinoma through the inhibition of chemotherapeutic-induced autophagy. PLoS ONE. 2015;10(5):e0121538. doi: 10.1371/journal.pone.0121538.

10. Kim SM, Oh EY, Lee JH, Nam D, Lee SG, Lee J, Kim SH, Shim BS, Ahn KS. Brassinin combined with capsaicin enhances apoptotic and anti-metastatic effects in PC-3 human prostate cancer cells. Phytother Res. 2015;29(11):1828-1836. doi: 10.1002/ptr.5478.

11. Bort A, Spinola E, Rodriguez-Henche N, Diaz-Laviada I. Capsaicin exerts synergistic antitumor effect with sorafenib in hepatocellular carcinoma cells through AMPK activation. Oncotarget. 2017;8(50):87684-87698. doi: 10.18632/oncotarget.21196.

12. Hers I, Vincent EE, Tavaré JM. Akt signalling in health and disease. Cell signal. 2011; 23(10): 1515-27. doi: 10.1016/j.cellsig.2011.05.004.

13. Chalhoub N, Baker SJ. PTEN and the PIE-Kinase Pathway in Cancer. Annu. Rev. Pathol. 2009. 4(1), pp. 127-150. doi: 10.1146/annurev.pathol.4.110807.092311.

14. Drake CG, Sharma P, Gerritsen W. Metastatic castration-resistant prostate cancer: new therapies, novel combination strategies and implications for immunotherapy. Oncogene. 2014;33(43):50535064. doi: 10.1038/onc.2013.497. 
15. McKeage K. Docetaxel: a review of its use for the first-line treatment of advanced castrationresistant prostate cancer. Drugs. 2012;72(11):1559-1577. doi: 10.2165/11209660-00000000000000.

16. Yu L, Wu X, Chen M, Huang H, He Y, Wang H, Li D, Du Z, Zhang K, Goodin S, et al. The effects and mechanism of YK-4-279 in combination with docetaxel on prostate cancer. Int J Med Sci. 2017;14(4):356-366. doi: 10.7150/ijms.18382.

17. Dirican A, Atmaca H, Bozkurt E, Erten C, Karaca B, Uslu R. Novel combination of docetaxel and thymoquinone induces synergistic cytotoxicity and apoptosis in DU-145 human prostate cancer cells by modulating PI3K-AKT pathway. Clin Transl Oncol. 2015;17(2):145-151. doi: 10.1007/s12094014-1206-6.

18. Yasumizu Y, Miyajima A, Kosaka T, Miyazaki Y, Kikuchi E, Oya M. Dual PI3K/mTOR inhibitor NVP-BEZ235 sensitizes docetaxel in castration resistant prostate cancer. J Urol. 2014;191(1):227234. doi: 10.1016/j.juro.2013.07.101.

19. Zheng L, Chen J, Ma Z, Liu W, Yang F, Yang Z, Wang K, Wang X, He D, Li L, et al. Capsaicin enhances anti-proliferation efficacy of pirarubicin via activating TRPV1 and inhibiting PCNA nuclear translocation in 5637 cells. Mol Med Rep. 2016;13(1):881-887. doi: 10.3892/mmr.2015.4623.

20. Clark R, Lee SH. Anticancer properties of capsaicin against human cancer. Anticancer Res. 2016;36(3):837-843.

21. Rollyson WD, Stover CA, Brown KC, Perry HE, Stevenson CD, McNees CA, Ball JG, Valentovic MA, Dasgupta P. Bioavailability of capsaicin and its implications for drug delivery. J Control Release. 2014;196:96-105. doi: 10.1016/j.jconrel.2014.09.027.

22. Nguyen HM, Vessella RL, Morrissey C, Brown LG, Coleman IM, Higano CS, Mostaghel EA, Zhang X, True LD, Lam HM, et al. LuCaP prostate cancer patient-derived xenografts reflect the molecular heterogeneity of advanced disease an-d serve as models for evaluating cancer therapeutics. Prostate. 2017;77(6):654-671. doi: 10.1002/pros.23313.

23. Wang Y, Lieberman R, Pan J, Zhang Q, Du M, Zhang P, Nevalainen M, Kohli M, Shenoy NK, Meng $\mathrm{H}$, et al. miR-375 induces docetaxel resistance in prostate cancer by targeting SEC23A and YAP1. Mol Cancer. 2016;15(1):70. doi: 10.1186/s12943-016-0556-9.

24. Zhang Y, Jin Z, Zhou H, Ou X, Xu Y, Li H, Liu C, Li B. Suppression of prostate cancer progression by cancer cell stemness inhibitor napabucasin. Cancer Med. 2016;5(6):1251-1258. doi: 10.1002/cam4.675. 